\title{
Cost-Effectiveness Analysis of Dacomitinib versus Gefitinib in the First-Line Treatment of EGFR-Positive Advanced or Metastatic Non-Small Cell Lung Cancer
}

\author{
Longfeng Zhang' \\ $\mathrm{Na} \mathrm{Li}{ }^{2}$ \\ Maobai Liu (D) ${ }^{2}$ \\ Bin Zheng ${ }^{2}$ \\ Zhijuan $\mathrm{Wu}^{3}$ \\ Hongfu Cai $\mathbb{D}^{2}$ \\ 'Department of Thoracic Oncology, \\ Fujian Provincial Cancer Hospital, Fujian \\ Medical University Cancer Hospital, \\ Fuzhou, Fujian Province, People's \\ Republic of China; ${ }^{2}$ Department of \\ Pharmacy, Fujian Medical University \\ Union Hospital, Fujian Medical University, \\ Fuzhou, Fujian Province, People's \\ Republic of China; ${ }^{3}$ Department of \\ Physiology and Pathophysiology, The \\ School of Basic Medical Sciences, Fujian \\ Medical University, Fuzhou, Fujian \\ Province, People's Republic of China
}

Background: The objective of the study was to evaluate the economics of dacomitinib and gefitinib in the first-line treatments for EGFR-positive advanced or metastatic non-small cell lung cancer (NSCLC) from a US payer perspective.

Methods: We developed the partition survival model to compare the lifetime cost and health outcomes of dacomitinib versus gefitinib. Transition probabilities were collected from the ARCHER 1050 trial. The model only considered the direct medical costs. Utility values were taken from published research.

Results: Compared to gefitinib, dacomitinib increased 0.706 QALY and the cost increased $\$ 232,359.32$. The incremental cost-effectiveness ratio (ICER) was $\$ 329,120.85$ per QALY in the base case. One-way sensitivity analysis showed that the cost of drugs and the utility had more influence on the results than other parameters. Probability sensitivity analysis reflected that the parameters had little effect on the results.

Conclusion: Dacomitinib could improve the health benefits and increase the overall costs. In this simulation, dacomitinib is not likely to be economical for first-line therapy of EGFRmutated NSCLC.

Keywords: dacomitinib, gefitinib, cost-effectiveness, non-small cell lung cancer

\section{Introduction}

Lung cancer has a high morbidity and mortality rate worldwide, most of which are non-small-cell lung cancer (NSCLC). ${ }^{1}$ The early symptoms of NSCLC are not obvious, and the diagnosis rate is low so that most patients miss the opportunity for surgery. ${ }^{2}$ Compared with the first representative epidermal growth factor receptor tyrosine kinase inhibitors (EGFR-TKIs), which are reversible inhibitors that selectively target EGFR, dacomitinib is a human EGFR family kinase activity and EGFR activating mutations some irreversible inhibitor. ${ }^{3,4}$ The Food and Drug Administration (FDA) has approved dacomitinib for the first-line treatment of EGFR-mutated metastatic NSCLC. ${ }^{5}$

A multicenter randomized double-blind controlled phase III clinical trial (ARCHER 1050) was conducted to compare the clinical efficacy and safety of the second-generation TKI dacomitinib with those of gefitinib in patients with EGFRpositive advanced or metastatic NSCLC. ${ }^{6}$ The trial demonstrated that dacomitinib has
Correspondence: Hongfu Cai; Maobai Liu Department of Pharmacy, Fujian Medical University Union Hospital, No. 29 Xinquan Road, Fuzhou, 35000I, Fujian

Province, People's Republic of China Email caihongfu3।@I26.com; liumaobai@I26.com 
significantly better progression-free survival (PFS) and overall survival (OS) than gefitinib, thus establishing the status of dacomitinib in the first-line treatment of NSCLC. The median PFS of patients in the dacomitinib and gefitinib groups were 14.7 and 9.2 months, the estimated 24-month PFS rates were $30.6 \%$ and $9.6 \%$, respectively. Recently updated clinical studies have shown that during a median follow-up of 31.3 months, $45.4 \%$ and $52.0 \%$ of patients in the dacomitinib and gefitinib groups died, respectively. The HR for OS was $0.760(0.582-0.993)$. The median OS rates of dacomitinib and gefitinib were 34.1 and 26.8 months, respectively. $^{7}$

The cost of drug treatment for NSCLC has caused a great economic burden. ${ }^{8}$ The cost of second-generation EGFRTKI dacomitinib drug is high, and no pharmacoeconomic research related to dacomitinib has been found. The present study aimed to evaluate the economics of dacomitinib and gefitinib in the first line treatment for patients with EGFRpositive NSCLC from a US payer perspective.

\section{Methods}

\section{Model Approach}

According to the disease development process and published pharmacoeconomic model of NSCLC, the partitioned survival model was established to simulate the disease process. The model analyzed the cost and output of the following strategies: the patients were treated with dacomitinib and gefitinib, respectively, and received dacomitinib $45 \mathrm{mg}$ or gefitinib $250 \mathrm{mg}$ daily until the disease progressed. The model structure includes three states, the patients were in a state of progression-free disease (PFD) when they were enrolled (Figure 1). The model was established by the TreeAge Pro 2018 software.
The assumption describing the survival benefits associated with first-line dacomitinib versus gefitinib was according to the outcomes from the trial. The study included stage IIIB/IV NSCLC diagnosed by histology or cytology, with at least one target lesion, and the target lesion had not received radiotherapy in the past. The tumor tissue was detected as EGFR mutation. ${ }^{6}$ In accordance with the National Comprehensive Cancer Network (NCCN) guidelines ${ }^{9}$ and random Phase III trial, patients treated with dacomitinib and gefitinib were assumed to continue treatment after the first or second-generation TKI treatment failed. ${ }^{6}$ Osimertinib was used for patients with EGFR T790M positive, and pemetrexed plus cisplatin chemotherapy was used for patients who were T790M negative. ${ }^{10}$ The cycle period was set to three weeks. The average age of the patients enrolled in the ARCHER 1050 study was 62 years. According to the experience of clinical experts, the model running time was set to ten years, which could fully cover the patient's full life cycle. The study only considered direct medical costs. Cost and utility value were discounted at a discount rate of $3 \%{ }^{11}$

The main evaluation indicator of the study was incremental cost-effectiveness ratio (ICER), and the main output indicators were quality-adjusted life years (QALYs, QALYs $=\mathrm{LYs} \times$ utility value), life years (LYs), and total cost. The willingness-to-pay (WTP) threshold was set to $\$ 100,000$ in accordance with the recommendations of Neumann. ${ }^{12}$ If ICER was less than the threshold, the dacomitinib group had a cost-effective advantage compared to the gefitinib group; if the ICER was greater than the threshold, the dacomitinib group had no cost-effective advantage.

\section{Clinical Parameters}

The transition probability was obtained from the KaplanMeier (K-M) curve reported by ARCHER 1050.6,7 The

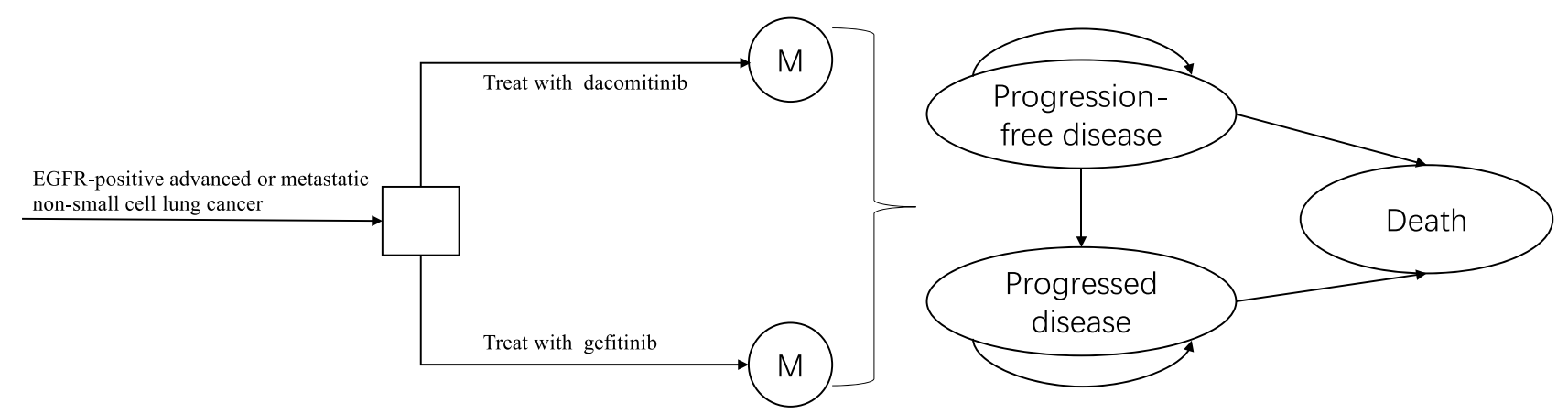

Figure I The Markov state transition model.

Notes: The Markov model simulates three states of NSCLC. At the end of each cycle, patients in the progression-free disease state may still in this state or in progressed disease state. Patients progressed disease state may still be in this state or enter death state. 
original PFS and OS information was obtained by GetData Graph Digitizer software 2.26. ${ }^{13}$ Two groups of the individual patient data of OS and PFS were reconstructed by R software (version 3.5.1; http://www.rproject.org). The transition probability in the model was calculated according to the survival data analysis process recommended by Hoyle et al. ${ }^{14}$ The fitted distribution functions included Weibull, log-logistic, exponential, log-normal and gamma. $^{15}$ According to Akaike information criterion (AIC) and Bayesian information criterion (BIC), combined with visual inspection, the optimal fitting distribution was judged and the distribution parameters of the fitting curve were obtained ${ }^{16}$ The results of PFS and OS fitted by Weibull distributions were more accurate (Figure 2). The Weibull distribution was used to fit and extrapolate the K-M survival curves reported in the original literature, and then the transition probability of each time $t$ was calculated. The survival probability at time $\mathrm{t}$ was $\mathrm{S}(\mathrm{t})=\exp \left(-\lambda \mathrm{t}^{\gamma}\right) .^{17} \lambda$ was the scale parameter, and $\gamma$ was the shape parameter. The model assumed that patients in PFD state only die due to natural mortality, so natural mortality was used as the transition probability from PFD to death. ${ }^{18}$ By comparing the median PFS and OS of ARCHER 1050 trial and curve simulation for internal verification, it showed that the fitting results are more accurate (Figure 2).

\section{Health Utilities}

Since no utility data were collected in ARCHER 1050 study, the utility values of each state came from the utility
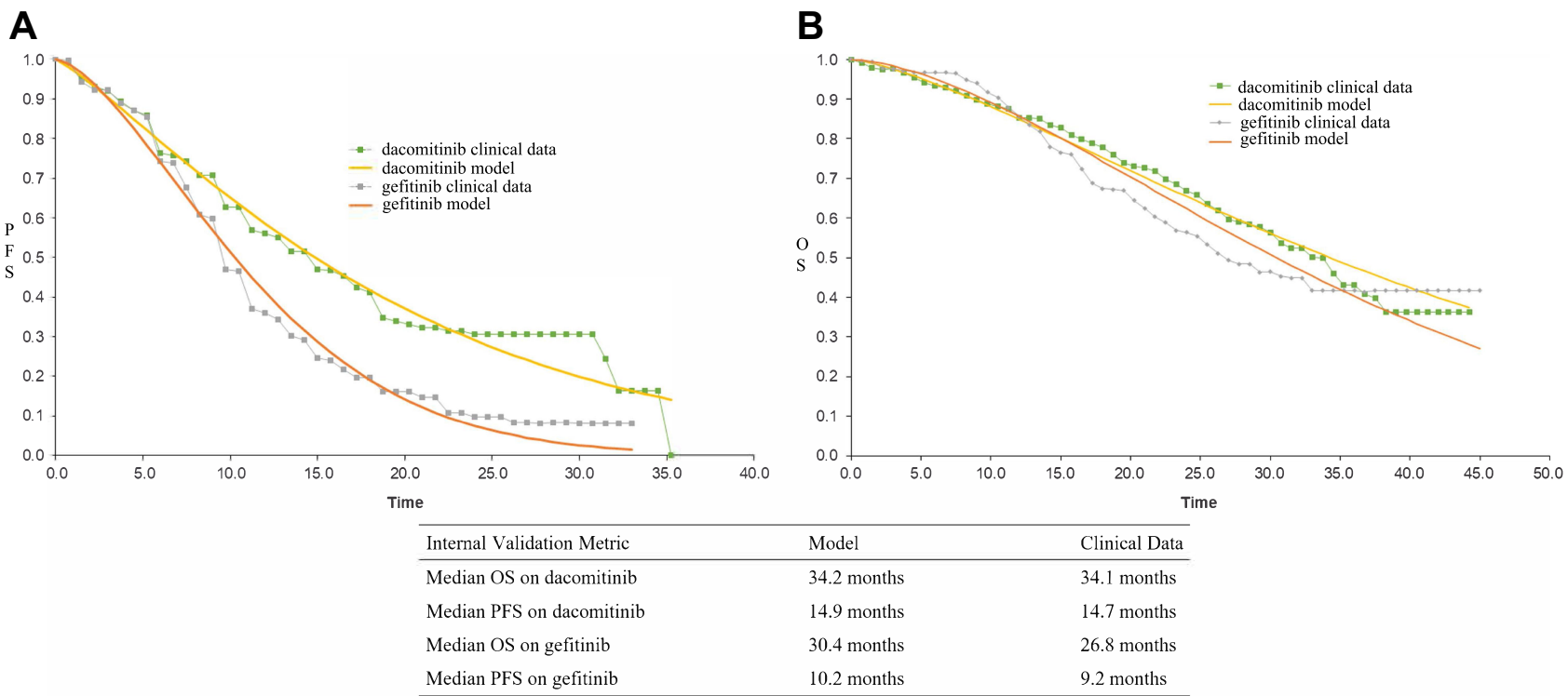

values of health state reported by Dansk. The utility of PFD state was 0.706 , and that of PD state was $0.565 .{ }^{19}$ The effect of adverse reactions with significant difference above grade 3 on utility value was considered. The calculation method was the utility of reducing adverse reactions multiplied by the incidence of adverse reactions. ${ }^{20}$ The incidences of diarrhea and rash/dermatitis in the dacomitinib group were $8 \%$ and $35 \%$, respectively, and those in the gefitinib group were $1 \%$ and $1 \%$, respectively. ${ }^{6}$

\section{Cost Estimates}

Direct medical costs included drug, routine follow up, salvage chemotherapy, supportive care, EGFR mutation testing and managing adverse events. ${ }^{21-23}$ The cost of dacomitinib, gefitinib, and osimertinib was based on the www.drugs.com database. $^{24}$ According to ARCHER 1050 study, the model assumed that $7.9 \%$ and $12.9 \%$ of the dacomitinib and gefitinib groups would receive osimertinib after progression, respectively. ${ }^{6}$ The routine dose of osimertinib was $80 \mathrm{mg}$ daily. The duration of pemetrexed plus cisplatin chemotherapy was assumed to be four cycles, and the pemetrexed monotherapy until disease progression. ${ }^{10}$ The model only considered serious adverse reactions above grade 3 . The incidences of adverse reactions above grade 3 for dacomitinib and gefitinib were $63 \%$ and $41 \%$, respectively. The cost of grades 3 and above adverse reaction was collected from other articles. ${ }^{6,25,26}$ The cost details are listed in Table 1 .

\begin{tabular}{lll}
\hline Internal Validation Metric & Model & Clinical Data \\
\hline Median OS on dacomitinib & 34.2 months & 34.1 months \\
Median PFS on dacomitinib & 14.9 months & 14.7 months \\
Median OS on gefitinib & 30.4 months & 26.8 months \\
Median PFS on gefitinib & 10.2 months & 9.2 months \\
\hline
\end{tabular}

Figure 2 The Kaplan-Meier curves of PFS and OS.

Notes: Weibull PFS cure fits to the Kaplan-Meier (K-M) trial data (A); Weibull OS curve fits to the K-M trial data (B). The smooth line represented the survival curve of Weibull distribution. The other lines indicated the survival curves in the ARCHER 1050 trial.

Abbreviations: OS, overall survival; PFS, progression-free survival. 
Table I Model Parameters

\begin{tabular}{|c|c|c|c|c|}
\hline \multirow[t]{2}{*}{ Variable } & \multirow[t]{2}{*}{ Baseline Value } & \multicolumn{2}{|c|}{ Range } & \multirow[t]{2}{*}{ Reference } \\
\hline & & Minimum & Maximum & \\
\hline \multicolumn{5}{|l|}{ Weibull PFS survival model } \\
\hline Dacomitinib & $\lambda=0.02688, \gamma=1.2036$ & - & - & {$[6,7]$} \\
\hline Gefitinib & $\lambda=0.01872, \gamma=1.5507$ & - & - & {$[6,7]$} \\
\hline \multicolumn{5}{|l|}{ Weibull OS survival model } \\
\hline Dacomitinib & $\lambda=0.005303, \gamma=1.3785$ & - & - & {$[6,7]$} \\
\hline Gefitinib & $\lambda=0.002802, \gamma=1.6133$ & - & - & {$[6,7]$} \\
\hline \multicolumn{5}{|l|}{ Utility } \\
\hline PFD & 0.706 & 0.620 & 0.815 & {$[19]$} \\
\hline $\mathrm{PD}$ & 0.565 & 0.470 & 0.688 & {$[19]$} \\
\hline Diarrhoea & -0.32 & -0.256 & -0.384 & [20] \\
\hline Rash or Dermatitis & -0.15 & -0.12 & -0.18 & [20] \\
\hline \multicolumn{5}{|l|}{ Cost (US \$) } \\
\hline \multicolumn{5}{|l|}{ Drug per day } \\
\hline Dacomitinib & 453.41 & 362.73 & 544.10 & {$[24]$} \\
\hline Gefitinib & 271.37 & 217.10 & 325.64 & [24] \\
\hline Osimertinib & 529.57 & 423.66 & 635.49 & [24] \\
\hline Pemetrexed plus cisplatin per cycle & 5,916 & 4,436 & 7,394 & {$[21]$} \\
\hline Pemetrexed maintenance per cycle & 5,797 & 2,898 & 8,695 & {$[21]$} \\
\hline Follow up per unit & 437 & 328 & 546 & {$[22]$} \\
\hline Supportive care per cycle & 8,770 & 6,578 & 10.963 & [22] \\
\hline EGFR mutation testing & 966 & 714 & 2,521 & [23] \\
\hline \multicolumn{5}{|l|}{ AEs cost } \\
\hline Diarrhea & 16,510 & 13,208 & 19,812 & {$[25]$} \\
\hline Rash or Dermatitis & $4,482.32$ & $4,156.95$ & $4,807.69$ & [26] \\
\hline
\end{tabular}

Abbreviations: PFD, progression-free disease; OS, overall survival; AE, adverse event; PD, progressed disease.

\section{Sensitivity Analysis}

One-way sensitivity analysis was performed to determine the influence of different parameters on ICER when changing within a certain range, and the results were presented in the form of tornado diagram. The upper and lower limits of the $95 \% \mathrm{CI}$ of the parameter value were derived from the literature. For some data that cannot get the $95 \% \mathrm{CI}$, the value of the parameter was floating by $\pm 20 \%$ on the basis value. In the probability sensitivity analysis, the parameters were set as random variables with specific distribution, and 10,000 Monte Carlo simulation was used to run the model to evaluate the overall robustness of the research results. It was assumed that the cost parameters have an gamma distribution, the incidence and health utility value parameters have beta distributions. ${ }^{27}$ The results of probability sensitivity analysis were presented by cost-effectiveness acceptable curve.

\section{Results}

The cumulative QALY was 1.507 QALY and 0.801 QALY in the dacomitinib group and the gefitinib group, respectively. The QALY in the dacomitinib group was 0.706 higher than those in the gefitinib group. The total cost of patients in the dacomitinib group was $\$ 361,331.16$, which was $\$ 232,359.32$ higher than that in the gefitinib group. Overall, dacomitinib demonstrated an ICER of $\$ 329,120.85$ per QALY compared with gefitinib. Dacomitinib also showed an ICER value higher than the WTP, which could be considered a uneconomic option. The results of basic analysis are shown in Table 2.

The tornado diagram is shown in Figure 3. The results of one-way sensitivity analysis showed that the most influential model parameters were the cost of dacomitinib, followed by the utility of PD, the proportion of second- 
Table 2 Cost-Effectiveness Analysis

\begin{tabular}{|l|c|c|c|c|}
\hline Strategies & Life Years & QALYs & $\begin{array}{c}\text { Total Costs } \\
\text { (US } \$ \text { ) }\end{array}$ & $\begin{array}{c}\text { ICER(US\$/QALY) } \\
\text { (Dacomitinib versus Gefitinib) }\end{array}$ \\
\hline Dacomitinib & 2.545 & 1.507 & $361,331.16$ & $329,120.85$ \\
Gefitinib & 1.247 & 0.801 & $128,971.84$ & \\
Incremental & 1.298 & 0.706 & $232,359.32$ & \\
(dacomitinib versus gefitinib) & & & & \\
\hline
\end{tabular}

Abbreviations: QALYs, quality-adjusted life-years; ICER, incremental cost-effectiveness ratio.

line osimertinib and the cost of gefitinib. Other model parameters, such as cost of follow-up, rash, and EGFR mutation testing, had little influence on the results.

The cost-effectiveness acceptability curve is shown in Figure 4. The probabilistic sensitivity analysis showed that the probability that dacomitinib has a cost-effectiveness advantage over gefitinib was $0 \%$ when the WTP threshold was $\$ 170,000$, the probability that dacomitinib has a cost- effectiveness advantage over gefitinib was $59.1 \%$ when the WTP threshold was $\$ 310,000$. The economic probability of dacomitinib increased with the increase in the average social WTP.

\section{Discussion}

The NCCN guidelines recommended osimertinib, erlotinib, afatinib, gefitinib, and dacomitinib as the first-line
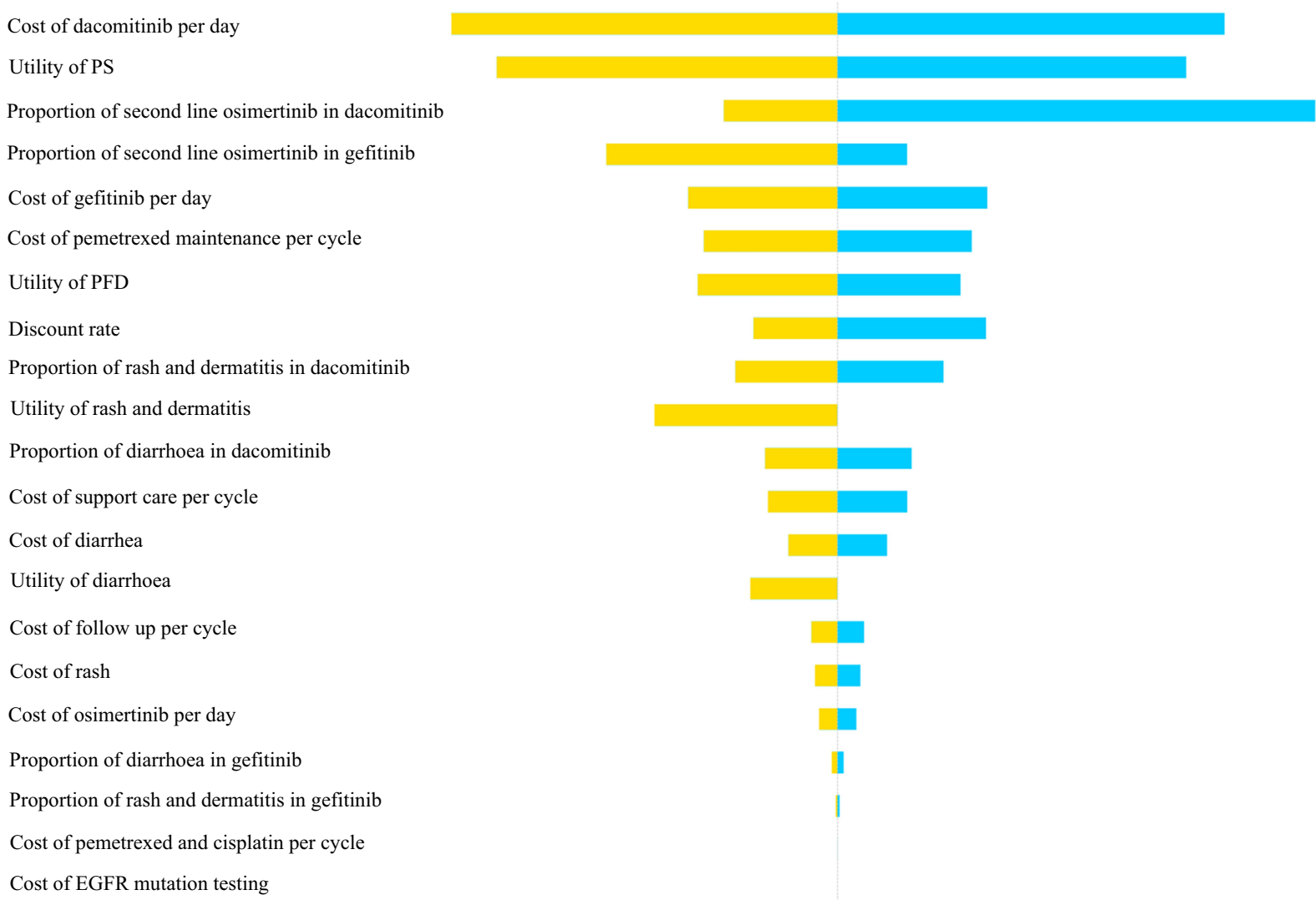

$2700000280000290000300000310000320000330000340000350000360000370000380000 \quad 390000 \quad 400000$

Figure 3 Tornado diagram.

Notes: The horizontal bar in the figure represents a parameter. The larger the span of the horizontal bar, the greater the influence of the parameter on the estimated value. Abbreviations: PD, progressed disease; PFD, progression-free disease. 


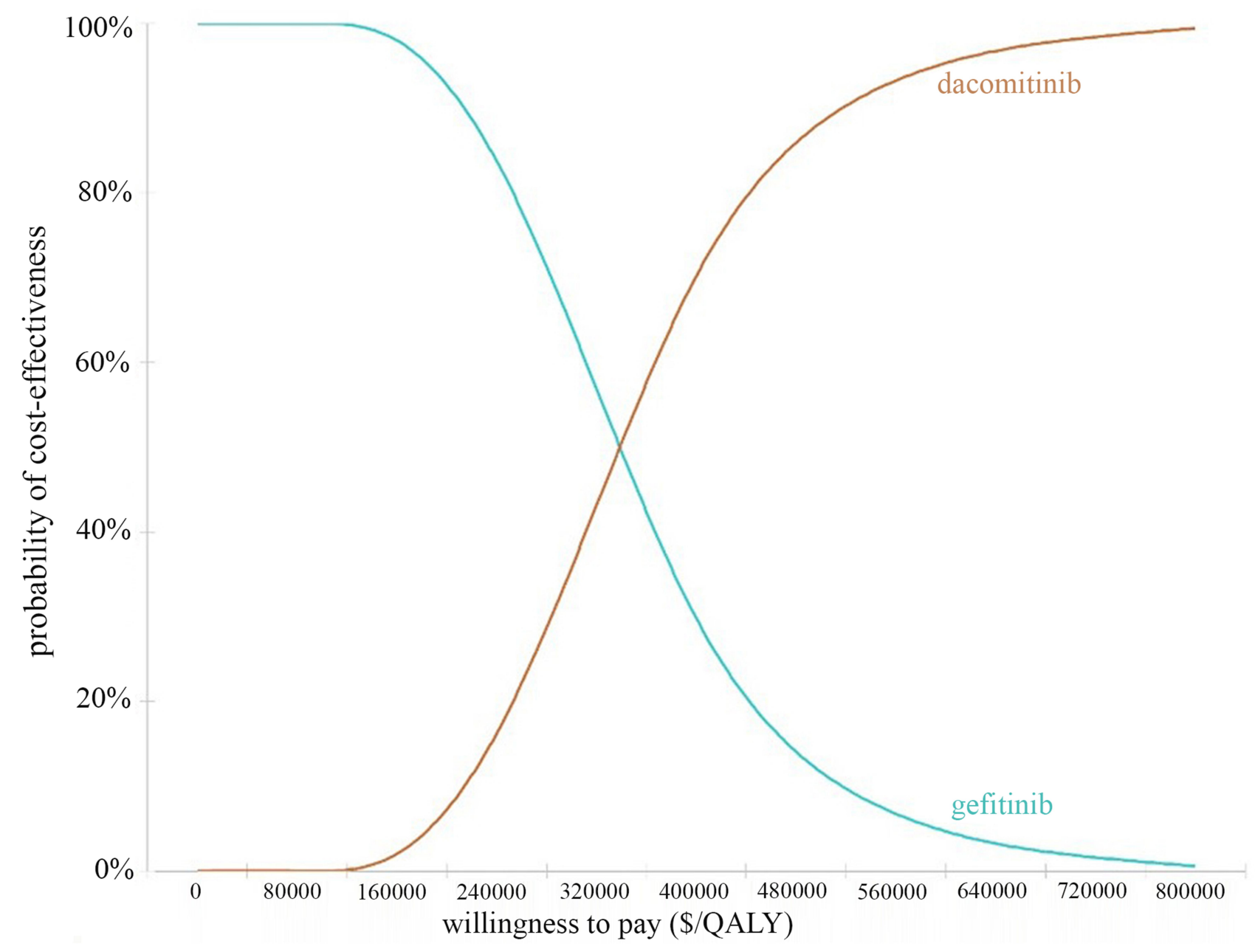

Figure 4 Cost-effectiveness acceptability curves (CEAC).

Note: CEAC shows the percentage of cost-effectiveness of each treatment at different thresholds.

treatment for EGFR sensitive mutations. ${ }^{9}$ Relevant pharmacoeconomic studies on osimertinib, erlotinib, afatinib, and gefitinib as the first-line treatment of NSCLC were found. Aguiar et $\mathrm{al}^{28}$ developed a Markov model based on the clinical data of the FLAURA clinical trial ${ }^{29}$ and evaluated the cost-effectiveness of osimertinib in the first-line treatment of patients with EGFR-mutant NSCLC. The study hypothesized that the clinical outcomes of afatinib were the same as those of gefitinib or erlotinib. In the US, the lifetime cost of osimertinib was $\$ 333,334$, and the lifetime utility gain was 2.122 QALY. In comparison to erlotinib, gefitinib and afatinib, the osimertinib ICERs were $\$ 226,527, \$ 231,123$, and $\$ 219,874$, respectively. The first-line treatment of osimertinib was not economical. $\mathrm{Wu}$ et $\mathrm{al}^{30}$ developed a Markov model to compare the economics of osimertinib with that of gefitinib or erlotinib as the first-line treatment of NSCLC. Osimertinib could provide more health benefits, and its mean cost and QALY as first-line treatment were $\$ 511,415$ and 2.316 , respectively. Compared with gefitinib or erlotinib, osimertinib was not a favorable first-line treatment based on American public payer's perspective. Ting et $\mathrm{al}^{31}$ developed a Markov model on the basis of the data from a large phase III clinical trial of EURTAC and LUX-Lung 3 to compare the cost-effectiveness of erlotinib and afatinib as first-line treatment of advanced EGFR-mutant NSCLC. Erlotinib had an ICER of $\$ 61,809 /$ QALY. Erlotinib was the preferred first-line treatment for advanced EGFR mutation-positive NSCLC in the United States. However, these studies did not involve the second-generation TKIs, and economic comparison between the second-generation TKIs and the first and third-generation TKIs was not clear.

To our knowledge, this study was the first to evaluate the economics of dacomitinib for NSCLC through economic modeling methods and latest evidence. The secondgeneration TKI dacomitinib has achieved significant 
clinical benefits. This study used a partitioned survival model to compare the cost-effectiveness of dacomitinib and gefitinib on the basis of the clinical data of ARCHER 1050. Dacomitinib had an ICER higher than the WTP of $\$ 100,000$, which was not economical at the current price. The price of dacomitinib was an important determinant of its economy.

The study did not compare the cost-effectiveness of the second and third generation TKIs. The population included in FLAURA and ARCHER 1050 was heterogeneous, and no head-to-head studies comparing dacomitinib with osimertinib are available. The ARCHER 1050 study did not include patients with central nervous system (CNS) metastasis, and it did not provide data on the treatment of patients with CNS metastases with dacomitinib. The present study was not applied to patients with CNS metastasis.

There are some deficiencies in the study. First, the currently analyzed data may need to be updated with the release of future data because the trials are still in progress. Second, the parameter distribution was used to fit and extrapolate the K-M survival curves of PFS and OS reported in the ARCHER 1050 trial. Although the result was verified, it could still lead to the uncertainty of the model output. Third, different chemotherapy regimens may be given when the disease progressed. The proportion of second-line treatment using osimertinib was referred to the data of clinical trials, which may be inconsistent with the real world. Despite these limitations, sensitivity analysis showed that the parameters did not affect the economic results. Each parameter in the model did not affect the final result.

\section{Conclusion}

When the WTP threshold is $\$ 100,000 / \$ 150,000$ per QALY, there is no cost-effective advantage of dacomitinib as a first-line treatment for patients with locally advanced or metastatic NSCLC with EGFR mutations.

\section{Acknowledgment}

This work was supported by Fujian Provincial Natural Science Foundation (grant number. 2017J01816 \& 2018Y9037), Fujian Provincial Health Commission (grant number 2019-CX-17), and National Natural Science Foundation of China (grant number 71804025).

\section{Disclosure}

The authors declare that they have no conflicts of interest.

\section{References}

1. Bray F, Ferlay J, Soerjomataram I, Siegel RL, Torre LA, Jemal A. Global cancer statistics 2018: GLOBOCAN estimates of incidence and mortality worldwide for 36 cancers in 185 countries. CA Cancer $J$ Clin. 2018;68(6):394-424. doi:10.3322/caac.21492

2. William WN, Lin HY, Lee JJ, Lippman SM, Roth JA, Kim ES. Revisiting stage IIIB and IV non-small cell lung cancer: analysis of the surveillance, epidemiology, and end results data. Chest. 2009;136 (3):701-709. doi:10.1378/chest.08-2968

3. Solca F, Dahl G, Zoephel A, et al. Target binding properties and cellular activity of afatinib (BIBW 2992), an irreversible ErbB family blocker. J Pharmacol Exp Ther. 2012;343(2):342-350. doi:10.1124/ jpet.112.197756

4. Engelman JA, Zejnullahu K, Gale CM, et al. PF00299804, an irreversible pan-ERBB inhibitor, is effective in lung cancer models with EGFR and ERBB2 mutations that are resistant to gefitinib. Cancer Res. 2007;67(24):11924-11932. doi:10.1158/0008-5472.CAN-07-1885

5. U.S. Food \& Drug Administration. FDA approves dacomitinib for metastatic non-small cell lung cancer. Available from: https://www. fda.gov/drugs/drug-approvals-and-databases/fda-approves-dacomitinib -metastatic-non-small-cell-lung-cancer-0. Accessed August 10, 2020.

6. Wu YL, Cheng Y, Zhou X, et al. Dacomitinib versus gefitinib as first-line treatment for patients with EGFR-mutation-positive non-small-cell lung cancer (ARCHER 1050): a randomised, open-label, Phase 3 trial. Lancet Oncol. 2017;18(11):1454-1466. doi:10.1016/S1470-2045(17)30608-3

7. Mok TS, Cheng Y, Zhou X, et al. Improvement in overall survival in a randomized study that compared dacomitinib with gefitinib in patients with advanced non-small-cell lung cancer and EGFR-activating mutations. J Clin Oncol. 2018;36(22):2244-2250. doi:10.1200/JCO.2018.78.7994

8. Wang $\mathrm{H}$, Naghavi $\mathrm{M}$, Allen $\mathrm{C}$, et al. Global, regional, and national life expectancy, all-cause mortality, and cause-specific mortality for 249 causes of death, 1980-2015: a systematic analysis for the Global Burden of Disease Study 2015. Lancet. 2016;388(10053):1459-1544.

9. National Comprehensive Cancer Network. NCCN Clinical Practice Guidelines in Oncology (NCCN Guidelines), Nonsmall Cell Lung Cancer, Version 6. 2020.

10. Mok TS, Wu YL, Ahn MJ, et al. Osimertinib or platinum-pemetrexed in EGFR T790M-positive lung cancer. $N$ Engl $J$ Med. 2017;376 (7):629-640. doi:10.1056/NEJMoa1612674

11. Sanders GD, Neumann PJ, Basu A, et al. Recommendations for conduct, methodological practices, and reporting of cost-effectiveness analyses: second panel on cost-effectiveness in health and medicine. JAMA. 2016;316(10):1093-1103. doi:10.1001/jama.2016.12195

12. Neumann PJ, Cohen JT, Weinstein MC. Updating costeffectiveness-the curious resilience of the $\$ 50,000$-per-QALY threshold. $N$ Engl $J$ Med. 2014;371(9):796-797. doi:10.1056/ NEJMp1405158

13. Wan X, Zhang Y, Tan C, Zeng X, Peng L. First-line nivolumab plus ipilimumab vs sunitinib for metastatic renal cell carcinoma: a cost-effectiveness analysis. JAMA Oncol. 2019;5(4):491-496. doi:10.1001/jamaoncol.2018.7086

14. Hoyle MW, Henley W. Improved curve fits to summary survival data: application to economic evaluation of health technologies. BMC Med Res Methodol. 2011;11:139. doi:10.1186/1471-2288-11-139

15. Ishak KJ, Kreif N, Benedict A, Muszbek N. Overview of parametric survival analysis for health-economic applications. Pharmacoeconomics. 2013;31(8):663-675. doi:10.1007/s40273-013-0064-3

16. Williams C, Lewsey JD, Mackay DF, Briggs AH. Estimation of survival probabilities for use in cost-effectiveness analyses: a comparison of a multi-state modeling survival analysis approach with partitioned survival and markov decision-analytic modeling. Med Decis Making. 2017;37(4):427-439. doi:10.1177/ 0272989X16670617 
17. Diaby V, Adunlin G, Montero AJ. Survival modeling for the estimation of transition probabilities in model-based economic evaluations in the absence of individual patient data: a tutorial. Pharmacoeconomics. 2014;32(2):101-108. doi:10.1007/s40273-0130123-9

18. Arias E. United States life tables, 2017. Natl Vital Stat Rep. 2019;68 (7): $1-66$.

19. Dansk V, Large S, Bertranou E, Bodnar C, Dyer M, Ryan JA. Review of health state utility values used in UK nice appraisals in advanced NSCLC. Value Health. 2016;19(7):A745-745A. doi:10.1016/j. jval.2016.09.2278

20. Nafees B, Lloyd AJ, Dewilde S, Rajan N, Lorenzo M. Health state utilities in non-small cell lung cancer: an international study. Asia Pac J Clin Oncol. 2017;13(5):e195-195,e203. doi:10.1111/ ajco. 12477

21. Handorf EA, McElligott S, Vachani A, et al. Cost effectiveness of personalized therapy for first-line treatment of stage IV and recurrent incurable adenocarcinoma of the lung. J Oncol Pract. 2012;8 (5):267-274. doi:10.1200/JOP.2011.000502

22. Klein R, Wielage R, Muehlenbein C, et al. Cost-effectiveness of pemetrexed as first-line maintenance therapy for advanced nonsquamous non-small cell lung cancer. $J$ Thorac Oncol. 2010;5 (8):1263-1272. doi:10.1097/JTO.0b013e3181e15d16

23. Sands J, Li Q, Hornberger J. Urine circulating-tumor DNA (ctDNA) detection of acquired EGFR T790M mutation in non-small-cell lung cancer: an outcomes and total cost-of-care analysis. Lung Cancer. 2017;110:19-25. doi:10.1016/j.lungcan.2017.05.014

24. The Drugs.com Database. Drug Price Information. Available from: https://www.drugs.com/price-guide/vizimpro.20200805. Accessed May 20, 2021.
25. Wan X, Luo X, Tan C, Zeng X, Zhang Y, Peng L. First-line atezolizumab in addition to bevacizumab plus chemotherapy for metastatic, nonsquamous non-small cell lung cancer: a United States-based cost-effectiveness analysis. Cancer. 2019;125 (20):3526-3534. doi:10.1002/cncr.32368

26. Kumar G, Woods B, Hess LM, et al. Cost-effectiveness of first-line induction and maintenance treatment sequences in non-squamous non-small cell lung cancer (NSCLC) in the U.S. Lung Cancer. 2015;89(3):294-300. doi:10.1016/j.lungcan.2015.05.020

27. Briggs AH, Weinstein MC, Fenwick EA, Karnon J, Sculpher MJ, Paltiel AD. Model parameter estimation and uncertainty analysis: a report of the ISPOR-SMDM modeling good research practices task force working group-6. Med Decis Making. 2012;32 (5):722-732. doi:10.1177/0272989X12458348

28. Aguiar PN, Haaland B, Park W, San Tan P, Del Giglio A, de Lima Lopes G. Cost-effectiveness of osimertinib in the first-line treatment of patients with EGFR-mutated advanced non-small cell lung cancer. JAMA Oncol. 2018;4(8):1080-1084. doi:10.1001/jamaoncol.2018.1395

29. Soria JC, Ohe Y, Vansteenkiste J, et al. Osimertinib in untreated EGFR-mutated advanced non-small-cell lung cancer. $N$ Engl J Med. 2018;378(2):113-125. doi:10.1056/NEJMoa1713137

30. Wu B, Gu X, Zhang Q, Xie F. Cost-effectiveness of osimertinib in treating newly diagnosed, advanced EGFR-mutation-positive non-small cell lung cancer. Oncologist. 2019;24(3):349-357. doi:10.1634/theoncologist.2018-0150

31. Ting J, Ho P T, Xiang P, Sugay A, Abdel-Sattar M, Wilson L. Costeffectiveness and value of information of erlotinib, afatinib, and cisplatin-pemetrexed for first-line treatment of advanced EGFR mutation-positive non-small-cell lung cancer in the United States. Value Health. 2015;18(6):774-782. doi:10.1016/j.jval.2015.04.008

\section{Publish your work in this journal}

Cancer Management and Research is an international, peer-reviewed open access journal focusing on cancer research and the optimal use of preventative and integrated treatment interventions to achieve improved outcomes, enhanced survival and quality of life for the cancer patient.
The manuscript management system is completely online and includes a very quick and fair peer-review system, which is all easy to use. Visit http://www.dovepress.com/testimonials.php to read real quotes from published authors. 